Rev. Elev. Méd. vét. Pays trop., 1973, 26 (4) : 75 a - 98 a

\title{
Biochimie et élevage au Sénégal
}

\author{
par D. FRIOT (*) et H. CALVET (*)
}

\begin{abstract}
RESUME
Le travail présenté s'efforce d'établir des normes biochimiques par 12 composants du sang des bovins au Sénégal. A partir de 24605 analyses sont déterminées les valeurs de l'hématocrite, hémoglobine, protéınes totales, urée, phosphore, calcium, magnésium, sodium, potassium, cuivre, fer et zinc. L'effet de la région, de la saison, de l'âge, du sexe et de la race est étudié pour chaque élément. Les résultats sont comparés aux taux moyens européens et à ceux obtenus en expérience d'embouche au Sénégal.
\end{abstract}

Durant ces vingt-cinq dernières années la chimie biologique a fait l'objet d'un développement considérable. Chaque jour de nouvelles techniques, tout particulièrement dans le domaine de l'enzymologie permettent de suivre, de façon de plus en plus intime, les processus normaux de la vie et leurs déviations qui conduisent aux maladies.

La clinique et la sémiologie humaine font depuis longtemps déjà un large usage des investigations biochimiques pour préciser ou nuancer un diagnostic, suivre une évolution morbide, ou plus récemment essayer de prévoir l'avenir pathologique des individus par établissement de "profils biochimiques» périodiques suivant les techniques américaines du «check up » dont le succès va croissant auprès dü public.

A leur tour, les vétérinaires et les techniciens de l'élevage ont pressenti le rôle important que pouvait jouer la biochimie dans le développement et l'intensification des productions animales.

Les applications pratiques sont certes encore peu nombreuses en regard des possibilités nouvelles révélées tous les jours par la recherche. Mais on peut envisager qu'avec la multiplication des laboratoires et des techniciens spécialisés dans ce domaine, avec l'intensification et l'industrialisation de plus en plus poussée de productions animales, «l'examen par sondage du profil biochimique d'un élevage apparaîtra dans les prochaines années aussi indispensable que la pesée des animaux ou la mesure de leur consommation d'aliment » (10).

Au Sénégal et particulièrement au Laboratoire national de Recherches vétérinaires de Dakar, les dosages biochimiques ont contribué pour une large part à un certain nombre de travaux visant à une meilleure connaissance de la physiologie des bovins tropicaux, à des études concernant leur nutrition, à des recherches sur les polycarences minérales dont ils sont victimes dans certaines zones d'élevage.

Les études se sont déroulées au Laboratoire ou dans les diverses stations de recherches du Sénégal - Ferme de Sangalkam, C.R.A. de Bambey, C.R.Z. de Dara. Plus récemment l'achat et l'équipement d'un véhicule laboratoire a permis la réalisation d'enquêtes beaucoup plus larges sur les troupeaux transhumants du Nord Sénégal ou sur les animaux sédentaires du Sine Saloum.

(*) I.E.M.V.T., Laboratoire National de 1'Elevage, B.P. n' 2057, Dakar-Hann, Sénégal. 
Les éléments biologiques objets de ces études ont varié en fonction des programmes. C'est ainsi qu'à l'étude de l'urémie des bovins tropicaux avec les mécanismes de son excrétion ou de sa réabsorption au niveau du rein ont succédé des recherches sur les protéines totales, leurs variations quantitatives ou qualitatives en fonction de l'âge, du sexe et de la saison (12-13-1415-16).

Le métabolisme de l'eau a cnsuite été abordé avec la mesure des espaces hydriques par dosage simultané des espaces de diffusion de l'antipyrine, du sulfocyanure de sodium, du bleu evans chez des zébus et des taurins entretenus dans des zones climatiques différentes (Sangalkam - Dara - Bouaké en Côte d'Ivoire) (17).

La biochimie avec en particulier le dosage des éléments minéraux plasmatiques a contribué à élucider l'étiologie et la pathogénie de la grave maladie enzootique qui sévissait sur les troupeaux du nord Sénégal (4).

Le développement du botulisme dans ces régions est apparu en effet comme étant favorisé par une hypophosphorose plasmatique associée à une carence en calcium et en cuivre (11).

Au cours des études sur les effets de l'aflatoxine sur les vaches laitières et sur les jeunes nourris à la mamelle, les tests biochimiques de l'exploration hépatique et tout particulièrement le dosage des transaminases TGO se sont avérés très efficaces pour déceler précocement l'intoxication avant l'apparition de tout autre symptôme.

La biochimie a encore joué un rôle important en nutrition et particulièrement pour l'établissement de rations d'embouche intensive. En effet, le dosage systématique des éléments énergétiques et azotés produits au niveau du rumen à la suite de l'administration d'une ration permet de préjuger efficacement de sa valeur et d'y apporter les modifications utiles pour en obtenir le meilleur rendement. En définitive et dans les années antérieures, la chimie biologique a donc été l'instrument indispensable de nombreuses recherches en physiologie, physiopathologie et nutrition.

Le travail, objet de cette note poursuit un objectif différent, et sans doute plus ambitieux. Il vise en effet, en traitant statistiquement un volume important de données, à établir un " profil biochimique " aussi précis et aussi vaste que possible du troupeau sénégalais en équilibre avec son milieu. Les éléments étudiés sont encore relativement restreints et portent, dans un premier temps, sur 12 composants du sang qui sont:

- Hémoglobine, protéines totales, urée, hématocrite, phosphore;

- calcium, magnésium, sodium, potassium, cuivre, zinc et fer.

Nous allons envisager chacun de ces éléments en indiquant succinctement les méthodes de dosage utilisées.

\section{Hématocrite}

L'hématocrite correspond à une mesure simple consistant en la séparation par centrifugation des globules et du plasma de sang hépariné introduit dans des tubes spéciaux, les tubes de Wintrobe. L'hématocrite des animaux a une composante individuelle essentielle dotée d'une certaine héritabilité, mais l'état général de l'animal intervient également et en particulier son niveau de nutrition, son équilibre hydrique et son taux de globules rouges.

\section{Hémoglobine}

L'hémoglobine constitue la matière colorée du sang composée d'une protéine, la globine et d'un groupement prosthétique, la protohématine. La teneur en hémoglobine diminue d'une façon plus ou moins notable chez tous les animaux.

La méthode de dosage utilisée comporte la dilution du sang total, recueilli sur héparine, dans le réactif de Drabkin et la mesure au photocolorimètre de la coloration obtenue à $530 \mathrm{~m} \mu$ ). 


\section{Protéines totales}

Elles sont dosées par la méthode photo colorimétrique de Gornall et lues à la longueur d'onde de $540 \mathrm{~m} \mu$.

La protéinémie sanguine participe à une homéostasie rigoureuse de l'individu. Les taux ne s'abaissent que dans les hyponutritions confirmées et lors de maladies graves. Chez le veau, il semble que l'examen des protéines sanguines constitue un excellent moyen pour déterminer l'évolution de la santé après la naissance. STAPLES et ses collaborateurs estiment qu'une protéinémie inférieure à 20 p. 100 s'observe chez 80 p. 100 des veaux destinés à mourir de maladie dans les mois qui suivent.

Plus intéressant encore que le taux des protéines totales sont les taux d'albumines et de globulines. La plupart des maladies infectieuses ou parasitaires se traduisent en effet par une diminution sensible du taux des albumines dont le niveau suit l'évolution de la maladie pour se reconstituer lors de la guérison.

\section{L'urémie}

Le taux de l'urémie chez les ruminants est soumis à de grandes fluctuations. Il varie en en effet d'un moment à l'autre de la journée, et d'un jour à l'autre. Des conclusions ne peuvent résulter que d'un grand nombre de mesures et sont alors liées d'abord à l'importance des apports protéiques de la ration et ensuite à la notion d'efficacité protéique c'est-à-dire le rapport entre l'azote protéique ingéré et le gain de poids.

LABOUCHE a étudié sur un troupeau de Sangalkam les variations moyennes au cours de deux périodes d'égale durée mai-septembre et septembre-janvier limitées par des valeurs minimales. Les maximums s'observent en juillet-août et octobre. Le facteur alimentaire exerce alors une influence prépondérante et en particulier le rapport azote/cellulose de la ration.

La méthode de dosage de l'urémie utilisée est la méthode à l'uréase avec coloration par le réactif de Nessler et lecture à $400 \mathrm{~m} \mu$.

\section{Phosphore sanguin}

Le dosage du phosphore inorganique présente des difficultés de plusieurs ordres.

Dans le sérum prélevé, on observe en effet une augmentation du $P$ inorganique avec le temps résultant de l'hydrolyse du phosphore à partir des combinaisons organiques. Pour certains auteurs, le meilleur moyen d'éviter cette source d'erreur est l'adjonction de l'acide trichloracétique en solution à 5 p. 100. Au laboratoire, les dosages du $\mathrm{P}$ sont effectués sur place et le plus rapidement possible après la récolte du sang dans le camion laboratoire conduit à proximité du lieu de prélèvement.

Une autre difficulté réside dans les grandes fluctuations individuelles et journalières enregistrées sur cette donnée et qui semble essentiellement tenir à l'état d'excitation de l'animal au moment du prélèvement. Des essais conduits au Laboratoire ont, en effet, montré qu'il était possible de diminuer très sensiblement l'amplitude de ces fluctuations en prélevant sur des animaux tranquillisés au préalable, ou lorsque, par répétitions des prélèvements, on arrive à un état d'accoutumance de l'animal.

Encore plus que pour les autres données, il convient donc d'user d'une grande prudence dans l'interprétation des résultats concernant la phosphorémie. Le phosphore inorganique est dosé sur le sérum déféqué et coloré par le réactif de Misson. La lecture se fait à $240 \mathrm{~m} \mu$.

\section{Calcium, sodium, magnésium, cuivre et zinc}

Ces dosages sont effectués sur les sérums déféqués par spectrophotométrie de flamme utilisant le spectrophotomètre atomique de Perkin Elmer modèle 290. 
Pour chacun de ces éléments, l'analyse statistique va s'efforcer de déterminer les moyennes obtenues et d'étudier l'influence des sources de variations contrôlables qui sont la race, la saison, la zone d'élevage, l'âge, le sexe et les états pathologiques.

\section{- RESULTATS :}

Le nombre total d'analyses ayant servi à l'établissement de ce travail est de 24605.

Les prélèvements ont été effectués dans plusieurs régions du Sénégal: Ferlo, Casamance, Sine Saloum, région de Diourbel, région de Thiès, ferme de Sangalkam. Ils intéressent tantôt des zébus, tantôt des taurins de race NDama, tantôt des métis zébu NDama (Djakoré) de sexe mâle ou femelle. Au cours de la plupart des prélèvements, l'âge de l'individu est enregistré et les résultats sont présentés en fonction de 5 classes d'âge.

Enfin, pour les enquêtes dans le Ferlo et dans la zone arachidière, les interventions ont ont été pratiquées au cours des 3 principales saisons bioclimatiques annuelles qui interviennent sur l'alimentation des troupeaux : hivernage — post hivernage ou saison favorable — saison sèche.

Les résultats vont être présentés élément par élément en adoptant l'ordre suivant : hématocrite, hémoglobine, protéines totales, urémie et les éléments minéraux (phosphore, calcium, cuivre, zinc, fer, sodium, potassium, magnésium). A l'intérieur de chaque chapitre et chaque fois que cela est possible, on essaiera de déterminer l'effet de la région, de la saison, de l'âge, du sexe, de la race et, éventuellement, des facteurs pathologiques.

\section{HEMATOCRITE}

Le nombre des données est de 2199 et la moyenne générale de 39,1 $\pm 0,3$.

Ce chiffre est parfaitement conforme à celui qu'on admet pour les troupeaux européens. DUKES (9) fixe à 40 la normalité de l'hématocrite pour les bovins.

\section{Effet de la région:}

Les prélèvements proviennent de régions à climatologie et écologie différentes.

Il s'agit du Ferlo (Labgar-Dara) de la zone arachidière avec (Nioro-du-Rip, Birkelane, Fatick, Diourbel, Kaël, Sagata, Kébémer, Mecké, Khombole) de la région des Niayes avec Sangalkam. Ces résultats sont groupés dans le tableau $\mathrm{n}^{\circ} \mathrm{I}$.

\begin{tabular}{|c|c|c|}
\hline Région & $\mathbb{N}$ & $\begin{array}{l}x+\text { intervalle de } \\
\text { conflance à } 5 \text { p. } 100\end{array}$ \\
\hline Fer to Labgar & 942 & $38,8 \pm 0,4$ \\
\hline C.R.Z. Dara & 240 & $44,2 \pm 0,7$ \\
\hline $\begin{array}{l}\text { Zone arachi- } \\
\text { dière }\end{array}$ & 978 & $38,0 \pm 0,4$ \\
\hline $\begin{array}{l}\text { Sangalkam } \\
\text { (embouche) }\end{array}$ & 39 & $44,1 \pm 0,3$ \\
\hline
\end{tabular}
Dara.

On constate qu'il y a deux groupes distincts : Labgar — Zone arachidière et Sangalkam - 
Le dernier groupe comprend des animaux élevés en station et qui, de ce fait, ont une nourriture légèrement améliorée et surtout un abreuvement régulier et abondant.

Dans ce chapitre, il est donc possible que le mode d'entretien agisse en définitive plus que la localisation géographique.

Effet de la saison:

Les résultats sont présentés dans le tableau $\mathrm{n}^{\circ}$ II.

TABLEAU N*II

Effet de la saison

\begin{tabular}{|l|c|c|}
\hline \multicolumn{1}{|c|}{ Saison } & $\mathrm{N}$ & $\begin{array}{c}\mathrm{X} \pm \text { intervalle de } \\
\text { confiance a 5 p.100 }\end{array}$ \\
\hline Hivernage & 295 & $39,2 \pm 0,6$ \\
Post hivernage & 511 & $39,9 \pm 0,5$ \\
Saison sèche & 412 & $38,4 \pm 0,7$ \\
\hline
\end{tabular}

L'analyse de la variance montre qu'il n'y a pas de différence significative entre l'hivernage et le post hivernage. Par contre, les données de saison sèche diffèrent significativement de ce dernier groupe.

L'hématocrite en saison sèche est donc plus bas qu'au cours du reste de l'année.

Ceci confirme l'hypothèse empirique suivant laquelle l'hématocrite constituerait un témoin du bon ou mauvais état général des individus.

Effet de l'âge :

Les classes d'âges utilisées se répartissent ainsi :

$$
\begin{array}{lllll}
\mathrm{D} \cdot & \cdot & \cdot & \text { de } 6 \text { mois à } 24 \text { mois compris } \\
\mathrm{E} \cdot & \cdot & \cdot & \text { de } 2 \text { ans à } 4 \text { ans } & \\
\mathrm{F} . & \cdot & \cdot & \text { de } 4 \text { ans à } 6 \text { ans } & \\
\mathrm{G} \cdot & \cdot & \cdot & \text { de } 6 \text { ans à } 10 \text { ans } & \\
\mathrm{H} \cdot & \cdot & \cdot & \text { au-delà de } 10 \text { ans. }
\end{array}
$$

Les valeurs de l'hématocrite en fonction de ces classes sont présentées dans le tableau $\mathbf{n}^{\circ}$ III et dans le graphique $\mathrm{A}$.

TABLEAU $\mathrm{N}^{\circ}$ III

Hêmatocrite en fonction de 1 'âge

\begin{tabular}{|c|r|c|}
\hline Classe & N & $\begin{array}{c}\text { Valeur moyenne } \pm \text { intervalle } \\
\text { de confiance à } 5 \text { p.100 }\end{array}$ \\
\hline D & 85 & $38,0 \pm 1,3$ \\
E & 223 & $40,6 \pm 0,7$ \\
F & 152 & $37,5 \pm 0,9$ \\
G & 238 & $36,0 \pm 0,7$ \\
H & 67 & $35,8 \pm 1,4$ \\
\hline
\end{tabular}

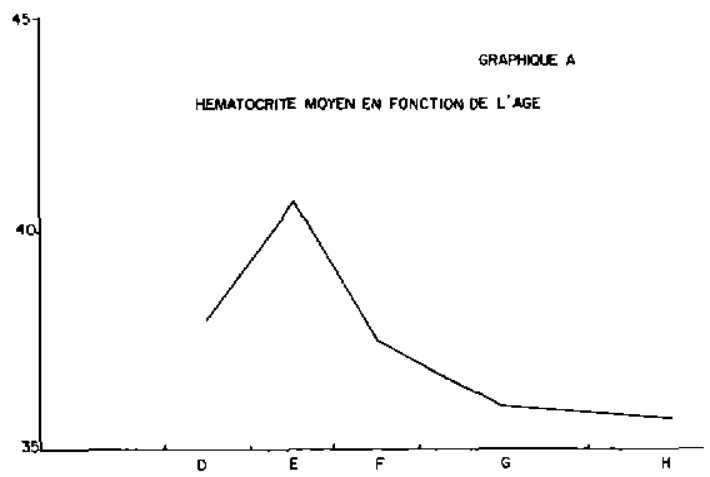


Seules les classes $\mathrm{G}$ et $\mathrm{H}$ ne sont pas significativement différentes comme il apparaît d'ailleurs sur la courbe.

L'hématocrite est donc maximal entre 2 et 4 ans et s'abaisse au-delà de 4 ans.

\section{Influence du sexe:}

On étudie 3 catégories d'individus, mâles, femelles et mâles castrés.

Les résultats sont portés dans le tableau suivant:

TABLEAU $N^{\circ}$ IV
Variation en fonction du sexe
\begin{tabular}{|l|r|r|}
\hline \multicolumn{1}{|c|}{ Sexe } & N & $\begin{array}{c}\text { Valeur moyenne } \pm \text { intervalle } \\
\text { de confiance à } 5 \text { p.100 }\end{array}$ \\
\hline Mâles & 45 & $38,5 \pm 1,5$ \\
Femelles & 369 & $38,3 \pm 0,6$ \\
Castrés & 39 & $42,4 \pm 1,8$ \\
\hline
\end{tabular}

L'analyse de variance (par comparaison orthogonale) montre une différence hautement significative entre le groupe mâles + femelles et les castrés, et ne révèle aucune différence entre les mâles et les femelles.

Les animaux sexuellement neutres ont donc un hématocrite plus élevé.

\section{Influence de l'espèce :}

Trois types d'animaux sont étudiés: les zébus de race Gobra, les taurins de race N'dama, et les métis de ces deux groupes dont l'appellation locale est Djakoré.

\begin{tabular}{|c|c|c|}
\hline \multicolumn{3}{|c|}{$\begin{array}{c}\text { Variations de l'hématocrite en fonction de } \\
\text { l'espêce et de la race }\end{array}$} \\
\hline $\begin{array}{l}\text { Espèce ou } \\
\text { race }\end{array}$ & $\mathrm{N}$ & $\begin{array}{l}\text { Valeur moyenne } \pm \text { intervalle } \\
\text { de confiance à } 5 \text { p.100 }\end{array}$ \\
\hline Zébus & 176 & $37,5 \pm 0,9$ \\
\hline Taurins & 98 & $34,7 \pm 1,2$ \\
\hline Djakorës & 38 & $37,7 \pm 2,2$ \\
\hline
\end{tabular}

L'analyse de la variance montre que le groupe Zébu + Djakoré est très significativement différent des taurins.

Les N'dama ont donc un hématocrite plus bas que celui des zébus et les Djakorés se comportent plus en zébus qu'en taurins.

En définitive, l'hématocrite moyen sur tous les dosages effectués au Sénégal est comparable aux valeurs considérées comme normales sur les troupeaux des régions tempérées.

Les facteurs de variations physiologiques sont, au Sénégal et par ordre d'importance, l'âge, le sexe et l'espèce.

\section{HEMOGLOBINE}

La moyenne générale du taux d'hémoglobine sur 1968 données est de 11,5 $\pm 0,1 \mathrm{~g} / 100$. 
Ce chiffre est légèrement inférieur mais très proche de celui des troupeaux européens pour lesquels DUKES donne la valeur de $12,0 \mathrm{~g} / 100$.

\section{Effet de la région:}

Les résultats sont groupés dans le tableau $\mathrm{n}^{\circ}$ VI.

TABLEAU N ${ }^{\circ} \mathrm{VI}$

Influence de la région

\begin{tabular}{|l|c|c|}
\hline \multicolumn{1}{|c|}{ Région } & $\mathrm{N}$ & $\begin{array}{c}\mathrm{X} \pm \text { intervalle de } \\
\text { confiance à 5 p.100 }\end{array}$ \\
\hline $\begin{array}{l}\text { Labgar } \\
\begin{array}{l}\text { Zone arachi- } \\
\text { dière } \\
\text { Sangalkam }\end{array}\end{array}$ & 944 & $12,0 \pm 0,1$ \\
& 38 & $11,1 \pm 0,1$ \\
\hline
\end{tabular}

La zone arachidière présente un taux d'hémoglobine différent des autres régions étudiées, avec des valeurs inférieures.

Effet de la saison:

Les résultats sont réunis dans le tableau $\mathrm{n}^{\circ}$ VII.

TABLEAU $N^{\circ} \mathrm{VII}$

Effet de la saison

\begin{tabular}{|l|c|c|}
\hline \multicolumn{1}{|c|}{ Saison } & $N$ & $\begin{array}{c}\overline{\mathrm{X}} \pm \text { intervalle de } \\
\text { confiance }\end{array}$ \\
\hline Hivernage & 234 & $11,3 \pm 0,2$ \\
Post hivernage & 441 & $10,9 \pm 0,2$ \\
Saison sèche & 311 & $11,2 \pm 0,2$ \\
\hline
\end{tabular}

Il existe une différence hautement significative entre le post hivernage et l'ensemble saison sèche et hivernage et pas de différence entre hivernage et saison sèche.

Paradoxalement, il semble donc que la saison favorable à l'animal, le post hivernage, soit celle où les valeurs de l'hémoglobine ont tendance à s'abaisser. Par ailleurs, nous avons constaté lors des expérimentations sur l'embouche intensive que le taux d'hémoglobine diminue au fur et à mesure que progresse l'engraissement.

Le post hivernage est la saison de reprise du poids des animaux et, comme au cours de l'embouche intensive, il semble que cette remise en état s'accompagne d'une diminution de l'hémoglobinémie.

Effet de l'âge:

Les résultats sont présentés en fonction des 5 classes d'âge, comme précédemment. G H.

Comme pour l'hématocrite, l'analyse de la variance montre qu'on peut regrouper les classes

Les valeurs de l'hémoglobine varient donc en fonction de l'âge. Elles passent par un maximum qui se situe entre 2 et 4 ans pour diminuer par la suite. 
TABLEÁU N"VIIT

Influence de 1 'âge sur 1 'hëmoglobinêmie

\begin{tabular}{|c|r|c|}
\hline $\begin{array}{l}\text { Classe } \\
\text { d'âge }\end{array}$ & N & $\begin{array}{c}\text { Valeur moyenne } \pm \text { intervalle } \\
\text { de confiance }\end{array}$ \\
\hline D & 85 & $11,7 \pm 0,4$ \\
E & 222 & $12,4 \pm 0,2$ \\
F & 152 & $11,7 \pm 0,3$ \\
G & 239 & $11,1 \pm 0,3$ \\
H & 66 & $11,2 \pm 0,5$ \\
\hline
\end{tabular}

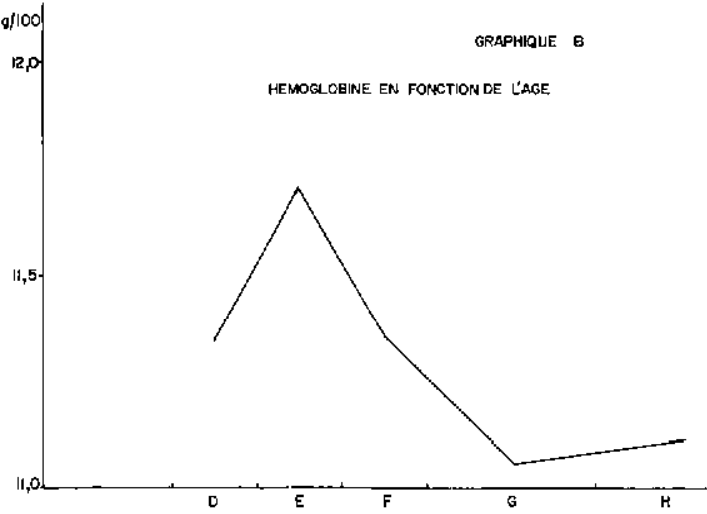

Les deux courbes de l'évolution des hématocrites et du taux d'hémoglobine en fonction de l'âge sont très comparables. Le coefficient de corrélation entre ces deux types de valeurs au même âge est significatif.

Ceci tendrait à prouver que le taux d'hémoglobine est davantage lié au nombre de globules qu'à leur charge unitaire en pigment.

\section{Influence du sexe:}

Les résultats sont groupés dans le tableau $\mathrm{n}^{0} \mathrm{IX}$.

L'analyse de variance par comparaison orthogonale montre qu'il existe une différence significative à 5 p. 100 entre l'ensemble mâles + femelles et l'ensemble des castrés, ces derniers ayant un taux d'hémoglobine plus élevé.

Ces résultats sont identiques à ceux obtenus pour l'hématocrite, ce qui paraît normal au vu de la corrélation positive hautement significative existant entre le taux d'hémoglobine et la valeur de l'hématocrite.

Les animaux castrés ont donc un taux d'hémoglobine plus élevé.

\section{Influence de l'espèce et de la race:} $\mathrm{n}^{\circ} \mathrm{X}$.

Les moyennes obtenues pour les différentes espèces et races sont présentées dans le tableau

Il existe une différence hautement significative entre les 3 types de bovins.

La différence existe entre les zébus et les taurins, les Djakoré se comportent en métis c'est-à-dire qu'ils se situent à mi-chemin entre les deux espèces, ne présentant, pour cette donnée, aucune différence pas plus à l'égard des taurins que des zébus.

TABLEAU $\mathrm{N}^{\circ} \mathrm{IX}$

Influence du sexe

\begin{tabular}{|l|r|c|}
\hline \multicolumn{1}{|c|}{ Sexe } & $\mathrm{N}$ & $\begin{array}{c}\overline{\mathrm{X}} \pm \text { interva1le de } \\
\text { confiance à 5 p.100 }\end{array}$ \\
\hline Mâles & 45 & $12,0 \pm 0,6$ \\
Femelles & 369 & $11,9 \pm 0,2$ \\
Castrês & 39 & $12,8 \pm 0,6$ \\
\hline
\end{tabular}

TABLEAU N* $\mathrm{X}$

Variations de 1'hémoglobine en fonction de l'espèce.

\begin{tabular}{|l|r|c|}
\hline \multicolumn{1}{|c|}{$\begin{array}{c}\text { Espèce ou } \\
\text { race }\end{array}$} & N & $\begin{array}{c}\text { Valeur moyenne } \pm \text { intervalle } \\
\text { de confiance à } 5.100\end{array}$ \\
\hline Zébus Gobra & 175 & $11,6 \pm 0,3$ \\
Taurins N'Dama & 98 & $10,5 \pm 0,4$ \\
Mêtis Djakoré & 38 & $11,1 \pm 0,6$ \\
\hline
\end{tabular}




\section{PROTEINES TOTALES}

La moyenne générale obtenue à partir de 2.195 analyses est de $85,4 \mathrm{~g} / \mathrm{l} \pm 0,8$. Cette valeur est légèrement supérieure aux normes européennes. Comme pour les données précédentes, nous allons analyser les facteurs de variation identifiables.

\section{Influence de la région:}

Il n'existe pas de différence significative entre Labgar et Sangalkam. Par contre, pour toutes les autres comparaisons deux à deux la différence est hautement significative.

\section{Influence de la saison:}

Il existe une différence significative entre l'hivernage et les autres saisons. Le post hivernage, saison favorable à l'élevage, donne les valeurs les plus basses, celles qui approchent donc le plus du taux admis comme norme européenne $(79 \mathrm{~g} / \mathrm{l})$. Il semble que les données voisines de ce dernier chiffre soient la signature d'un équilibre de l'animal avec son milieu.

TABLEAU $\mathrm{N}^{\circ} \mathrm{XI}$

Influence de la région

\begin{tabular}{|l|r|c|}
\hline \multicolumn{1}{|c|}{ Rëgion } & $N$ & $\begin{array}{c}\text { Valeur moyenne } \pm \text { intervalie } \\
\text { de confiance à } 5.100\end{array}$ \\
\hline Ferlo Labgar & 949 & $84,2 \pm 1,0$ \\
Ferlo Dara CRZ & 239 & $80,2 \pm 1,3$ \\
Niayes Sangalkam & 40 & $84,3 \pm 1,5$ \\
Zone arachidière & 967 & $87,9 \pm 1,4$ \\
\hline
\end{tabular}

TABLEAU $\mathrm{N}^{\bullet} \mathrm{XII}$

influence de la saison

\begin{tabular}{|c|c|c|}
\hline Saíson & $\mathrm{N}$ & $\begin{array}{l}\text { Valeur moyenne } \pm \text { intervalle } \\
\text { de confiance à } 5 \text { p.100 }\end{array}$ \\
\hline Hivernage & 291 & $101,3 \pm 3$ \\
\hline Post hivernage & 510 & $81,5 \pm 0,9$ \\
\hline Saison sèche & 405 & $81,9 \pm 1,9$ \\
\hline
\end{tabular}

\section{Influence de l'âge:}

L'analyse de la variance montre une différence significative entre les classes $D$ et $E$ et entre les classes $\mathrm{E}$ et $\mathrm{F}$; par contre, les classes $\mathrm{F}, \mathrm{G}$ et $\mathrm{H}$ ne diffèrent pas entre elles et peuvent être réunies en une classe unique de moyenne $91,6 \pm 1,7$.

La protéinémie augmente rapidement avec l'âge. Cependant les classes F, G, H groupant les animaux reproducteurs marquent un palier dans cette évolution. Il est possible que cette diminution relative de la protéinémie soit en rapport avec les besoins élevés en protéines de l'état de gestation puis de lactation.

TABLEAU $\mathrm{N}^{\bullet} \mathrm{XIII}$

Influence de 1 'âge

\begin{tabular}{|c|c|c|}
\hline Classe d'âge & N & $\begin{array}{l}\text { Valeur moyenne } \pm \text { intervalle } \\
\text { de confiance à } 5.100\end{array}$ \\
\hline D & 84 & $80,0 \pm 3,8$ \\
\hline $\mathrm{E}$ & 221 & $86,0 \pm 2,5$ \\
\hline $\mathrm{F}$ & 148 & $93,1 \pm 2,3$ \\
\hline $\mathbf{G}$ & 237 & $89,9 \pm 2,3$ \\
\hline $\mathrm{H}$ & 66 & $94,4 \pm 5,9$ \\
\hline
\end{tabular}

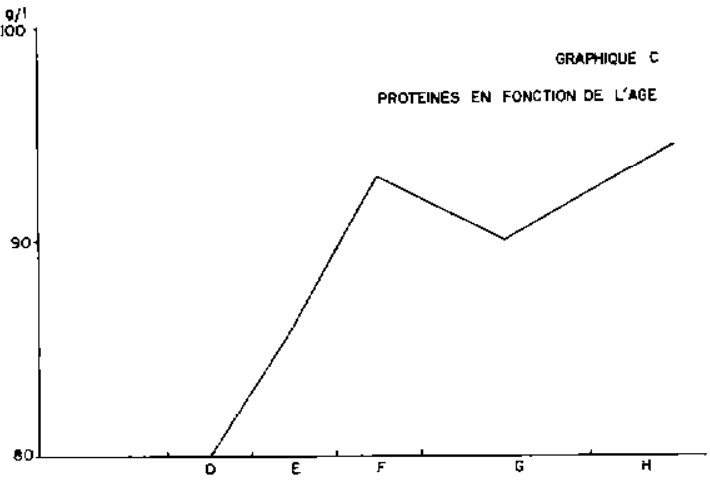




\section{Influence du sexe:}

Sur l'ensemble, il n'y a pas de différence significative entre ces 3 catégories d'animaux.

Le sexe n'influe donc pas significativement sur le taux des protéines sériques.

\section{Influence de l'espèce ou de la race:}

Il n'existe pas de différence entre les zébus et les taurins. Par contre, par rapport à ce groupe les métis sont significativement différents avec un taux de protéines plus bas.

TABLEAU N ${ }^{\circ} \mathrm{XIV}$

Influence du sexe

\begin{tabular}{|l|r|c|}
\hline \multicolumn{1}{|c|}{ Sexe } & N & $\begin{array}{r}\text { Valeur moyenne } \pm \text { interval1e } \\
\text { de confiance à 5 p.100 }\end{array}$ \\
\hline Femelles & 367 & $92,0 \pm 1,5$ \\
Mâles & 45 & $87,7 \pm 4,0$ \\
Castrës & 39 & $90,8 \pm 5,1$ \\
\hline
\end{tabular}

TABLEAU $\mathrm{N}^{\circ} \mathrm{XV}$

Influence de l'espèce ou de la race

\begin{tabular}{|l|r|c|}
\hline Espèce & N & $\begin{array}{r}\text { Valeur moyenne } \pm \text { intervalle } \\
\text { de confiance à } 5 \text { p.100 }\end{array}$ \\
\hline Zêbus & 172 & $85,9 \pm 3,9$ \\
Taurins & 97 & $82,9 \pm 3,2$ \\
Métis & 38 & $72,3 \pm 2,4$ \\
\hline
\end{tabular}

\section{UREMIE}

Le nombre d'analyses correspondant à cette donnée est de 1934. La valeur moyenne s'élève à $293 \pm 6 \mathrm{mg} / 1$.

Influence de la région:

Les trois régions présentent entre elles des différences hautement significatives.

La valeur augmente du Ferlo à Sangalkam avec la zone arachidière occupant une place intermédiaire.

A ce stade (urémie peu élevée), le taux de l'urée sanguine est essentiellement lié à l'importance des apports protéiques qui sont les plus faibles au Ferlo et les plus forts à Sangalkam, cette évolution correspond parfaitement aux données écologiques.

\section{Influence de la saison:}

Il existe une différence significative entre la saison sèche et la saison des pluies.

Par contre, la différence est hautement significative entre ces deux dernières saisons et le post hivernage.

L'explication de ces résultats fait appel, ici, à la notion d'efficacité protéique, qui est la meilleure lorsqu'il existe dans la ration un équilibre favorable entre l'apport azoté et énergétique;

TABLEAU $\mathbb{N}^{\circ} \mathrm{XVI}$

Influence de la rëgion

\begin{tabular}{|l|r|c|}
\hline Rëgion & N & $\begin{array}{r}\text { Yaleur moyenne } \pm \text { interva11e } \\
\text { de confiance à } 5 \text { p.100 }\end{array}$ \\
\hline Ferlo Labgar & 949 & $268 \pm 6$ \\
Zone arachidière & 947 & $313 \pm 9$ \\
Niayes Sangalkan & 38 & $393 \pm 29$ \\
\hline
\end{tabular}

TABLEAU $\mathrm{N}^{\circ} \mathrm{XVII}$

Influence de la saison

\begin{tabular}{|l|c|c|}
\hline \multicolumn{1}{|c|}{ Saison } & N & $\begin{array}{c}\text { Paleur moyenne } \pm \text { intervalle } \\
\text { de confiance à } 5 \text { p.100 }\end{array}$ \\
\hline Hivernage & 229 & $384 \pm 15$ \\
Post hivernage & 427 & $240 \pm 10$ \\
Saison sèche & 291 & $367 \pm 18$ \\
\hline
\end{tabular}


de plus, une bonne efficacité protéique se traduit par une urémie basse, conditions qui semblent se rencontrer dans le post hivernage.

Dans les deux autres saisons, soit en raison d'un apport excédentaire en azote (hivernage) soit au contraire en raison d'une insuffisance d'énergie (saison sèche) l'efficacité protéique diminue avec son corollaire: l'élévation du taux de l'urée.

Influence de l'âge:

TABLEAU $N^{\circ}$ XVIII
Inf luence de 1 'âge
\begin{tabular}{|c|r|c|}
\hline Classe d'âge & N & $\begin{array}{c}\text { Valeur moyenne } \pm \text { interval1e } \\
\text { de confiance a } 5 \text { p.100 }\end{array}$ \\
\hline D & $B 3$ & $300 \pm 23$ \\
E & 218 & $307 \pm 16$ \\
F & 146 & $309 \pm 23$ \\
G & 233 & $300 \pm 15$ \\
H & 66 & $286 \pm 29$ \\
\hline
\end{tabular}

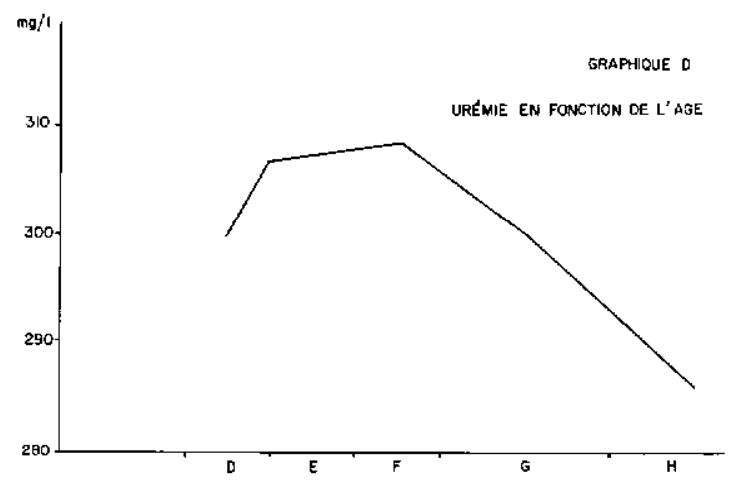

Il n'existe pas de différence significative entre les classes d'âge, mais on note tout de même une évolution comme en témoigne le graphique $\mathrm{D}$.

\section{Influence du sexe:}

Il n'existe pas de différence significative entre sexes.

\section{Influence de l'espèce :}

Il existe une différence hautement significative entre les 3 groupes.

Les zébus ont l'urémie la plus élevée, les taurins la plus basse, et les métis montrent une situation intermédiaire avec cependant une tendance à se rapprocher davantage des zébus que des taurins.

TABLEAU $N^{\circ} \mathrm{XIX}$

Urëmie en fonction du sexe

\begin{tabular}{|l|r|c|}
\hline \multicolumn{1}{|c|}{ Sexe } & N & $\begin{array}{r}\text { Valeur moyenne } \pm \text { interval1e } \\
\text { de conflance a 5 p.100 }\end{array}$ \\
\hline Femelles & 372 & $259 \pm 8$ \\
Mâles & 45 & $265 \pm 21$ \\
Castrés & 39 & $270 \pm 22$ \\
\hline
\end{tabular}

TABLEAU $N^{*} \mathrm{XX}$

Influence de l'espèce

\begin{tabular}{|l|r|c|}
\hline \multicolumn{1}{|c|}{ Espèce } & N & $\begin{array}{c}\text { Valeur moyenne } \pm \text { intervalle } \\
\text { de confiance }\end{array}$ \\
\hline Zébus & 160 & $408 \pm 26$ \\
Taurins & 93 & $301 \pm 24$ \\
Mêt1s & 38 & $358 \pm 42$ \\
\hline
\end{tabular}

\section{PHOSPHORE}

Le nombre total de dosages concernant cet élément est de 3033.

La moyenne générale en résultant est de $62,1 \pm 0,9 \mathrm{mg} / \mathrm{l}$.

\section{Influence de la région:}

On peut regrouper les données concernant la Casamance, le C.R.Z. de Dara et la zone 
TABLEAU $\mathrm{N}^{\circ} \mathrm{XXI}$

Influence de la région

\begin{tabular}{|l|c|c|}
\hline \multicolumn{1}{|c|}{ Région } & N & $\begin{array}{c}\text { Valeur moyenne } \pm \text { intervalle } \\
\text { de confiance à 5 p.100 }\end{array}$ \\
\hline Ferlo & 1478 & $50,1 \pm 1,4$ \\
Casamance & 163 & $70,7 \pm 1,6$ \\
CRz Dara & 240 & $74,5 \pm 1,4$ \\
Zone arach1dière & 985 & $73,3 \pm 1,1$ \\
Sangalkam & 129 & $60,7 \pm 1,3$ \\
pâturages & & $90,3 \pm 2,4$ \\
Sangalkam & 38 & \\
\hline
\end{tabular}

arachidière pour lesquelles les valeurs de la phosphorémie ne sont pas significativement différentes.

Ce groupe par contre diffère du Ferlo, de Sangalkam pâturage, et très nettement de Sangalkam embouche.

A ce niveau (phosphorémie moyenne), les taux de phosphore inorganique sérique sont sous la dépendance étroite des apports de phosphore par la ration et l'eau d'abreuvement.

Ces apports sont les plus faibles dans le Ferlo, et les plus élevés dans les rations d'embouche à Sangalkam qui contenaient en moyenne 3 à 4 p. 100 de suppléments minéraux (phosphate bicalcique).

\section{Influence de la saison:}

Les données concernant ce chapitre et pour les deux grandes régions, Ferlo et Zone arachidière où ont été effectués les prélèvements, ne sont pas cohérentes.

Nous devons étudier successivement l'influence de la saison sur la phosphorémie au Ferlo et en zone arachidière.

Les valeurs indiquées au tableau XXII présentent entre elles une différence hautement significative.

Dans le tableau XXIII aussi les différences entre les 3 saisons sont hautement significatives.

Nous nous trouvons donc en présence de deux régions où l'évolution de la phosphorémie de l'hivernage à la saison sèche se produit en sens inverse.

Dans le Ferlo, le phosphore sérique diminue de l'hivernage en saison sèche. Dans la zone arachidière il augmente.

Cette incohérence peut résulter de conditions écologiques et alimentaires très différentes pour les troupeaux de l'une et l'autre région.

TABLEAU $\mathrm{N}^{\mathrm{X} X \mathrm{XI}}$

Influence de la saison ar la phosphorémie au Ferlo

\begin{tabular}{|l|r|c|}
\hline \multicolumn{1}{|c|}{ Saison } & N & $\begin{array}{c}\text { Valeur moyenne } \pm \text { intervalle } \\
\text { de confiance a } 5.100\end{array}$ \\
\hline Hivernage & 252 & $64,9 \pm 2,0$ \\
Post hivernage & 0 & $50,7 \pm 2,0$ \\
Salson sèche & 269 & \\
\hline
\end{tabular}

TABLEAU $\mathrm{N}^{\circ} \mathrm{XXIII}$

Influence de la saison sur la phosphorêmie en zone arachidière

\begin{tabular}{|l|c|c|}
\hline \multicolumn{1}{|c|}{ Saison } & $\mathbb{N}$ & $\begin{array}{c}\text { Valeur moyenne } \pm \text { intervalle } \\
\text { de confiance à 5.100 }\end{array}$ \\
\hline Hivernage & 234 & $64,6 \pm 2,2$ \\
Post hivernage & 441 & $73,7 \pm 1,4$ \\
Saison sèche & 310 & $79,3 \pm 2,2$ \\
\hline
\end{tabular}


Dans le Ferlo, et tout au long de l'année, la base de l'alimentation des troupeaux est constituée par les pâturages de savane où dominent les graminées peu riches en phosphore surtout lorsqu'elles sont transformées en paille de saison sèche. En zone arachidière par contre, les animaux fréquentent au cours de l'année deux types différents de pâturage.

Pendant la saison des pluies, période de mise en culture des champs, les troupeaux sont refoulés sur des zones pâturables plus ou moins distantes des villages où ils se trouvent concentrés. Leur alimentation est alors essentiellement à base de graminées. Dès la récolte, les animaux reviennent autour du village, exploitent les résidus de récolte et passent la saison sèche sur les jachères. L'apport alimentaire essentiel provient alors des repousses arbustives qui envahissent ces jachères, avec comme dominante les repousses de Guiera senegalensis dont les analyses révèlent une richesse en éléments minéraux et en phosphore bien supérieure à celle des graminées.

Le taux élevé du phosphore en saison sèche et dans la zone arachidière pourrait donc tenir à la consommation habituelle de cette espèce végétale.

\section{Influence de l'âge :}

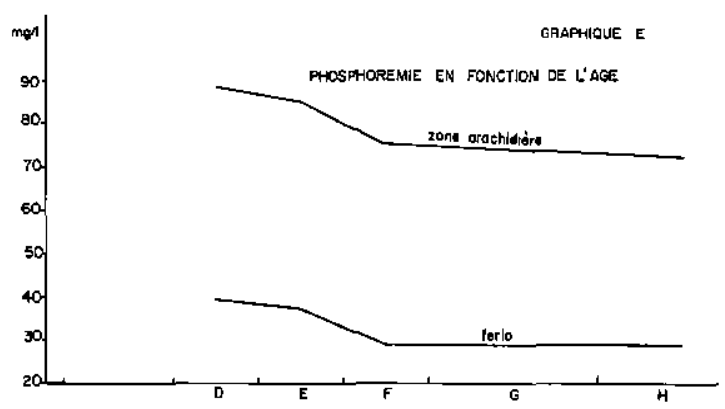

TABLEAU $\mathrm{N}^{\circ} \mathrm{XXIV}$

Effet de l'âge sur la phosphorémie du Ferlo

\begin{tabular}{|c|r|c|}
\hline Classe d'âge & $\mathrm{N}$ & $\begin{array}{c}\text { Valeur moyenne } \pm \text { intervalle } \\
\text { de confiance } 5 \text { p.100 }\end{array}$ \\
\hline D & 36 & $38,7 \pm 3,9$ \\
E & 131 & $37,1 \pm 1,7$ \\
F & 98 & $29,3 \pm 2,7$ \\
G & 140 & $28,8 \pm 2,0$ \\
H & 51 & $29,2 \pm 4,2$ \\
\hline
\end{tabular}

TABLEAU $\mathrm{N}^{\circ} \mathrm{XXV}$

Effet de l'âge sur la phosphorémie en zone arachidière

\begin{tabular}{|c|r|c|}
\hline Classe d'âge & N & $\begin{array}{c}\text { Valeur moyenne } \pm \text { intervalle } \\
\text { de confiance a } 5.100\end{array}$ \\
\hline D & 48 & $88,3 \pm 6,5$ \\
E & 92 & $84,6 \pm 3,5$ \\
F & 55 & $74,8 \pm 6,0$ \\
G & 100 & $73,7 \pm 3,4$ \\
H & 15 & $71,9 \pm 11,2$ \\
\hline
\end{tabular}

Pour cet effet et comme précédemment en raison des valeurs différentes dans le Ferlo et la zone arachidière, il faut étudier séparément les deux régions.

On peut regrouper les classes $\mathrm{D}$ et $\mathrm{E}$ et les classes $\mathrm{F}, \mathrm{G}, \mathrm{H}$. La phosphorémie des jeunes est donc différente de celle des adultes (tableau XXIV).

On peut regrouper les classes comme précédemment $D$ et $E$ d'une part, $F, G, H$ d'autre part (tableau XXV).

Situées à des niveaux moyens très différents, les évolutions de la phosphorémie en fonction de l'âge dans le Ferlo et en zone arachidière sont parfaitement parallèles. 


\section{Influence du sexe:}

L'ensemble mâles + castrés diffèrent significativement des femelles; mâles et castrés sont comparables.

\section{Influence de l'espèce :}

Il y a différence significative entre les 3 groupes. Les taurins Ndama diffèrent de manière hautement significative de l'ensemble zébus-métis. Les métis ont une phosphorémie non différente de celle des zébus. Les taurins ont une phosphorémie plus faible.

TABLEAU N XXVI

Influence du sexe sur la phosphorëmie du Ferlo

\begin{tabular}{|l|r|c|}
\hline \multicolumn{1}{|c|}{ Sexe } & N & $\begin{array}{r}\text { Valeur moyenne } \pm \text { intervalle } \\
\text { de confiance à } 5 \text { p.100 }\end{array}$ \\
\hline Femelles & 372 & $30,6 \pm 1,3$ \\
Mâles & 45 & $37,9 \pm 2,5$ \\
Castrés & 39 & $39,9 \pm 2,8$ \\
\hline
\end{tabular}

TABLEAU N`XXVII

Phosphorémie et espèce

\begin{tabular}{|l|r|c|}
\hline Espèce & N & $\begin{array}{c}\text { Valeur moyenne } \pm \text { intervalle } \\
\text { de confiance à } 5 \text { p.100 }\end{array}$ \\
\hline Zëbus & 239 & $80,6 \pm 2,6$ \\
Métis & 38 & $81,5 \pm 6,3$ \\
Taurins & 226 & $66,7 \pm 1,8$ \\
\hline
\end{tabular}

\section{CALCIUM}

La calcémie moyenne obtenue à partir de 2652 analyses est de $102,6 \pm 0,6 \mathrm{mg} / \mathrm{l}$. Elle est supérieure à la valeur de $91 \mathrm{mg} / 1$ donnée par DUKES comme norme européenne.

\section{Influence de la région:}

Les différences entre régions sont hautement significatives. En Casamance, zone à climat favorable, et à Sangalkam pendant l'expérience d'embouche la calcémie est identique aux $91 \mathrm{mg} / 1$ de DUKES. Dans les autres régions les calcémies sont supérieures. Il semble donc qu'il y ait déséquilibre dans les zones moins favorisées.

Influence de la saison:

Les différences entre saisons sont hautement significatives. La calcémie du post hivernage est très significativement différente de l'ensemble hivernage - saison sèche. La différence entre saison sèche et hivernage est faible mais significative. Remarquons que c'est en post hivernage, saison la plus favorable, que la calcémie est la plus proche des $91 \mathrm{mg} / 1 \mathrm{de}$ la norme européenne de DUKES.

TABLEAU $\mathrm{N}^{\bullet} \mathrm{XXVIII}$

Influence de la région

\begin{tabular}{|l|r|c|}
\hline \multicolumn{1}{|c|}{ Région } & $N$ & $\begin{array}{c}\text { Valeur moyenne } \pm \text { intervalle } \\
\text { de confiance à 5 p.100 }\end{array}$ \\
\hline Ferlo & 1385 & $102,6 \pm 0,8$ \\
Casamance & 140 & $92,5 \pm 1,5$ \\
C.R.z. Dara & 240 & $96,5 \pm 0,9$ \\
Zone arachidière & 719 & $105,4 \pm 1,3$ \\
Sangalkam & 130 & $99,8 \pm 1,6$ \\
pâturages & & $90,8 \pm 1,3$ \\
Sangalkam & 38 & \\
\hline
\end{tabular}

TABLEAU $N^{\circ} \mathrm{XXIX}$

Influence de la saison

\begin{tabular}{|l|c|c|}
\hline \multicolumn{1}{|c|}{ Saison } & N & $\begin{array}{c}\text { Valeur moyenne } \pm \text { intervalle } \\
\text { de confiance à } 5 \text { p.100 }\end{array}$ \\
\hline Hivernage & 293 & $109,9 \pm 1,3$ \\
Post hivernage & 265 & $89,3 \pm 2,0$ \\
Saison sèche & 401 & $107,5 \pm 1,4$ \\
\hline
\end{tabular}




\section{Influence de l'âge:}

Comme pour le phosphore, nous pouvons regrouper les classes $\mathrm{D}$ et $\mathrm{E}$ d'un côté et les classes F, G, $\mathrm{H}$ d'un autre. La calcémie des jeunes diffère de celle des adultes et lui est supérieure. ensuite.

Nous constatons une baisse de la calcémie jusqu'à l'âge de 4-5 ans et une stabilisation

TABLEAU $\mathrm{N}^{\circ} \mathrm{XXX}$

Influence de l'âge

\begin{tabular}{|c|c|c|}
\hline Classe d'âge & $N$ & $\begin{array}{r}\text { Valeur moyenne } \begin{array}{c}\text { intervalle } \\
\text { de confiance à } 5,100\end{array} \\
\hline \text { D }\end{array}$ \\
E & 78 & $111,9 \pm 3,1$ \\
F & 149 & $109,1 \pm 1,5$ \\
G & 236 & $105,0 \pm 2,0$ \\
H & 65 & $104,8 \pm 1,9$ \\
\end{tabular}

GRAPAIOUE $F$

CALEEME EN FONCTION OE L'AEE

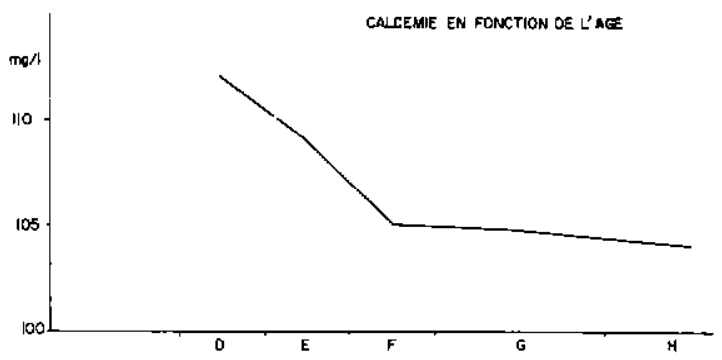

Influence du sexe:

Le sexe de l'animal influe sur la calcémie. Les mâles ne diffèrent pas des castrés mais les femelles ont une calcémie significativement différente de l'ensemble mâles - castrés.

Influence de l'espèce:

Les taurins N'dama ne diffèrent pas des métis Djakoré et les zébus sont significativement différents de l'ensemble taurins djakoré.

TABLEAU $\mathrm{N}^{\circ} \mathrm{XXXI}$

Influence du sexe

\begin{tabular}{|l|r|r|}
\hline \multicolumn{1}{|c|}{ Sexe } & N & $\begin{array}{r}\text { Valeur moyenne } \pm \text { intervalle } \\
\text { de confiance à } 5.100\end{array}$ \\
\hline Femelles & 359 & $102,3 \pm 1,2$ \\
Máles & 44 & $107,0 \pm 3,3$ \\
Castrés & 39 & $108,6 \pm 3,3$ \\
\hline
\end{tabular}

TABLEAU $\mathrm{N}^{\circ} \mathrm{XXXII}$

Influence de l'espèce

\begin{tabular}{|l|r|c|}
\hline Espèce & N & $\begin{array}{c}\text { Valeur moyenne } \\
\text { de confiance à 5 P.100 }\end{array}$ \\
\hline Zébus & 169 & $114,1 \pm 2,1$ \\
Métis & 38 & $107,6 \pm 3,1$ \\
Taurins & 94 & $109,3 \pm 2,9$ \\
\hline
\end{tabular}

\section{CUIVRE}

La cuprémie des bovins calculée sur 2791 analyses est de $0,60 \mathrm{mg} / 1 \pm 0,01$. Beaucoup d'auteurs considèrent que le seuil de la carence en cuivre se situe à $0,60 \mathrm{mg} / \mathrm{l}$, les teneurs normales variant de 0,75 à $1 \mathrm{mg} / \mathrm{l}$. Le taux moyen sénégalais est à la limite de la carence.

\section{Influence de la région:}

La cuprémie diffère suivant les régions. Remarquons que seule la zone arachidière a une cuprémie supérieure au seuil de carence. Il existe donc au Sénégal une carence en cuivre chez les bovins (tableau XXXIII).

Influence de la saison:

La cuprémie diffère suivant les saisons. La cuprémie de saison sèche ne diffère pas de celle de l'hivernage mais la cuprémie du post hivernage est très significativement différente de celle 
TABLEAU $\mathrm{N}^{a} \mathrm{XXXIII}$

Influence de la région

\begin{tabular}{|l|r|c|}
\hline \multicolumn{1}{|c|}{ Région } & N & $\begin{array}{c}\text { Valeur moyenne } \pm \text { intervalle } \\
\text { de confiance a 5 p.100 }\end{array}$ \\
\hline Ferlo & 1405 & $0,55 \pm 0,01$ \\
Casamance & 153 & $0,49 \pm 0,02$ \\
C.R.Z. Dara & 240 & $0,60 \pm 0,01$ \\
Zone arachidiêre & 953 & $0,69 \pm 0,01$ \\
Sangalkam & 40 & $0,59 \pm 0,02$ \\
\hline embouche & & \\
\hline
\end{tabular}

TABLEAU N ${ }^{\circ} \mathrm{XXXIV}$

Influence de la saison

\begin{tabular}{|l|c|c|}
\hline \multicolumn{1}{|c|}{ Saison } & $\mathrm{N}$ & $\begin{array}{c}\text { Valeur moyenne } \\
\text { de confiance intervalle }\end{array}$ p.100 \\
\hline Hivernage & 293 & $0,63 \pm 0,02$ \\
Post hivernage & 494 & $0,75 \pm 0,02$ \\
Saison sèche & 406 & $0,61 \pm 0,02$ \\
\hline
\end{tabular}

de l'ensemble hivernage - saison sèche. Le taux de cuivre sérique est alors plus élevé, ce qui contribue à un meilleur état général des animaux durant le post hivernage.

Influence de l'âge:

Il y a différence significative entre classes d'âge. La cuprémie diminue en fonction de l'âge. Nous pouvons regrouper $D$ et $E$ en un seul groupe ainsi que $F, G, H$. La cuprémie des jeunes est plus élevée que celle des adultes.

TABLEAU N ${ }^{\circ} \mathrm{XXXV}$

Influence de 1 'âge

\begin{tabular}{|c|r|c|}
\hline Classe d'âge & $N$ & \begin{tabular}{c} 
Valeur moyenne $\begin{array}{c}\text { intervalle } \\
\text { de confiance à } 5.100\end{array}$ \\
\hline D
\end{tabular} \\
E & 79 & $0,62 \pm 0,04$ \\
F & 150 & $0,58 \pm 0,02$ \\
G & 237 & $0,54 \pm 0,02$ \\
H & 65 & $0,54 \pm 0,02$ \\
& & $0,51 \pm 0,03$ \\
\hline
\end{tabular}

GRAPHIQUE $G$

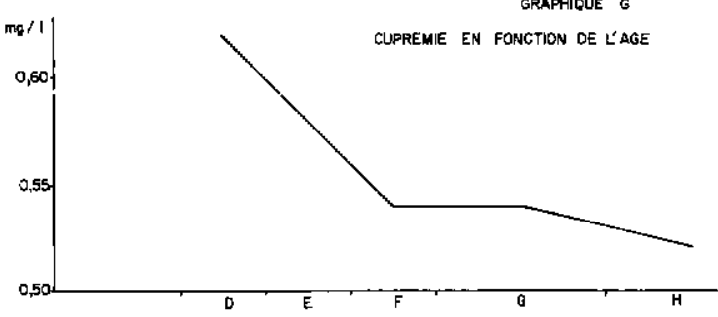

\section{Influence du sexe :}

L'analyse de variance montre qu'il n'existe pas de différence significative entre sexes mais la cuprémie chez les mâles et les castrés semble supérieure à celle des femelles.

\section{Influence de l'espèce:}

Il n'y a pas de différence entre la cuprémie des taurins N'dama et la cuprémie des métis Djakoré. La cuprémie chez les zébus est très significativement différente de celle de l'ensemble taurins mêtis.

\begin{tabular}{|c|c|c|}
\hline Sexe & $\mathrm{N}$ & $\begin{array}{l}\text { Valeur moyenne } \pm \text { intervalle } \\
\text { de confiance à } 5 \text { p. } 100\end{array}$ \\
\hline Feme1les & 361 & $0,51 \pm 0,01$ \\
\hline Mâles & 44 & $0,53 \pm 0,05$ \\
\hline Castrës & 39 & $0,53 \pm 0,04$ \\
\hline
\end{tabular}

TABLEAU $\mathrm{N}^{\bullet} \mathrm{XXXVII}$

Influence de $1^{\prime}$ espèce

\begin{tabular}{|l|r|c|}
\hline \multicolumn{1}{|c|}{ Espèce } & N & $\begin{array}{c}\text { Valeur moyenne } \pm \text { intervalle } \\
\text { de confiance à 5 p.100 }\end{array}$ \\
\hline Zébus & 173 & $0,68 \pm 0,02$ \\
Métis & 38 & $0,54 \pm 0,04$ \\
Taurins & 95 & $0,54 \pm 0,03$ \\
\hline
\end{tabular}




\section{ZINC}

La teneur en zinc sérique obtenue à partir de 1918 analyses est de 1,46 mg/l $\pm 0,03$. Ce chiffre paraît supérieur aux normes européennes qui se situent autour de 0,60 à $1 \mathrm{mg} / \mathrm{l}$.

\section{Influence de la région:}

Il existe des différences entre régions très importantes.

TABLEAU $\mathrm{N}^{\circ} \mathrm{XXXVIII}$

Influence de 1 a région

\begin{tabular}{|c|c|c|}
\hline Règlon & $\mathrm{N}$ & $\begin{array}{l}\text { Waleur moyenne } \pm \text { intervalle } \\
\text { de confiance a } 5 \text { p.100 }\end{array}$ \\
\hline Fer10 & 796 & $1,50 \pm 0,05$ \\
\hline Casamance & 91 & $2,23 \pm 0,14$ \\
\hline C.R.Z. Dara & 239 & $1,31 \pm 0,03$ \\
\hline Zone arachldlère & 754 & $1,39 \pm 0,03$ \\
\hline $\begin{array}{l}\text { Sangalkam } \\
\text { embouche }\end{array}$ & 38 & $1,04 \pm 0,04$ \\
\hline
\end{tabular}

Influence de la saison:

Les résultats obtenus sont contradictoires suivant les régions prospectées. Nous donnerons donc 2 tableaux correspondant à 2 régions différentes.

TABLEAU $\mathrm{N}^{\circ} \mathrm{XXXIX}$

Dara C.R.z. ceneur en zinc sêrique

\begin{tabular}{|l|r|c|}
\hline \multicolumn{1}{|c|}{ Saison } & N & $\begin{array}{c}\text { Valeur moyenne } \pm \text { intervalle } \\
\text { de conf iance a 5 p.100 }\end{array}$ \\
\hline Hivernage & 60 & $1,41 \pm 0,05$ \\
Post hivernage & 79 & $1,30 \pm 0,07$ \\
Saison sèche & 100 & $1,26 \pm 0,06$ \\
\hline
\end{tabular}

TABLEAU $\mathrm{N}^{\bullet} \mathrm{XL}$

Teneur en zinc de la zone arachidıère en fonction des saisons

\begin{tabular}{|l|c|c|}
\hline \multicolumn{1}{|c|}{ Saison } & N & $\begin{array}{c}\text { Valeur moyenne } \pm \text { intervalle } \\
\text { de confiance à 5 p.100 }\end{array}$ \\
\hline Hivernage & 228 & $1,09 \pm 0,03$ \\
Post hivernage & 409 & $1,49 \pm 0,04$ \\
Saison sèche & 117 & $1,65 \pm 0,07$ \\
\hline
\end{tabular}

Pour Dara il n'y a pas de différence entre post hivernage et saison sèche mais il y a une différence significative entre l'hivernage et l'ensemble post hivernage - saison sèche. Les teneurs en zinc sérique sont plus élevées en hivernage.

Il existe (tableau XL) des différences entre les saisons et les teneurs en zinc sont plus faibles en hivernage.

\section{Influence de l'âge:}

La teneur en zinc varie avec l'âge. Elle diminue en fonction de l'âge.

TABLEAU $\mathrm{N}^{\bullet} \mathrm{XLI}$

Influence de 1 'âge

\begin{tabular}{|c|r|c|}
\hline Classe d'âge & N & $\begin{array}{c}\text { Valeur moyenne } \pm \text { interval le } \\
\text { de confiance } 5 \text { P.100 }\end{array}$ \\
\hline D & 43 & $1,39 \pm 0,12$ \\
E & 154 & $1,31 \pm 0,05$ \\
F & 113 & $1,29 \pm 0,06$ \\
G & 169 & $1,25 \pm 0,05$ \\
H & 56 & $1,21 \pm 0,07$ \\
\hline
\end{tabular}

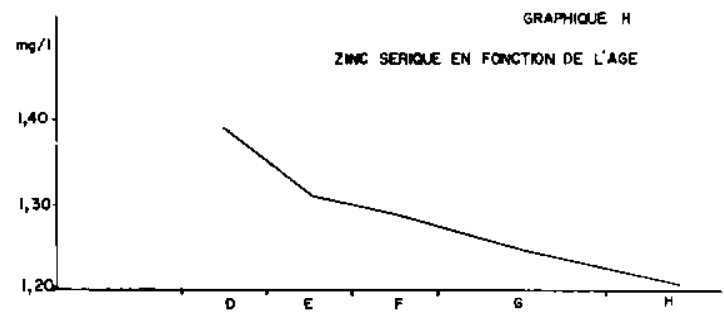


Influence du sexe:

La teneur en zinc du sérum est indépendante du sexe.

\section{Influence de l'espèce:}

L'analyse statistique par comparaisons orthogonales montre qu'il n'y a pas de différence entre métis et taurins et que la différence entre les zébus et l'ensemble métis - taurins est très significative.

TABLEAU $\mathrm{N}^{\bullet}$ XLII

Influence du sexe

\begin{tabular}{|l|r|c|}
\hline \multicolumn{1}{|c|}{ Sexe } & N & $\begin{array}{c}\text { Valeur moyenne } \\
\text { de confiance intervalle } 5 \text { p.100 }\end{array}$ \\
\hline Femelles & 351 & $1,22 \pm 0,04$ \\
Mâles & 35 & $1,21 \pm 0,08$ \\
Castrés & 39 & $1,18 \pm 0,07$ \\
\hline
\end{tabular}

TABLEAU $\mathrm{N}^{\circ}$ XLIII

Influence de $l^{\prime}$ 'espèce

\begin{tabular}{|l|l|c|}
\hline Espèce & N & $\begin{array}{c}\text { Valeur moyenne } \pm \text { intervalle } \\
\text { de confiance à } 5 \text { p.100 }\end{array}$ \\
\hline Zébus & 62 & $1,80 \pm 0,08$ \\
Métis & 12 & $1,52 \pm 0,25$ \\
Taurins & 43 & $1,46 \pm 0,12$ \\
\hline
\end{tabular}

\section{LE FER}

La moyenne générale sur 944 animaux est de $1,32 \mathrm{mg} / 1 \pm 0,03$.

Influence de la région:

La teneur en fer sérique est variable suivant les régions.

Influence de la saison:

La teneur en fer sérique est la même en hivernage et en post hivernage; cette teneur en saison sèche est différente de celle de l'ensemble hivernage post hivernage.

TABLEAU N* XLIV

Influence de la région

\begin{tabular}{|l|c|c|}
\hline \multicolumn{1}{|c|}{ Région } & $\mathrm{N}$ & $\begin{array}{c}\text { Valeur moyenne } \\
\text { de confiance interva11e } \\
5 \mathrm{p} .100\end{array}$ \\
\hline Ferlo & 370 & $1,17 \pm 0,04$ \\
Cagamance & 138 & $1,40 \pm 0,09$ \\
C.R.Z. Dara & 217 & $1,59 \pm 0,03$ \\
Zone arachidtère & 219 & $1,25 \pm 0,04$ \\
\hline
\end{tabular}

TABLEAU $\mathrm{N}^{\circ} \mathrm{XLV}$

Influence de la saison

\begin{tabular}{|l|r|c|}
\hline \multicolumn{1}{|c|}{ Saison } & N & $\begin{array}{r}\text { Valeur moyenne } \pm \text { intervalle } \\
\text { de confiance }\end{array}$ \\
\hline Hivernage & 51 & $1,63 \pm 0,06$ \\
Post hivernage & 66 & $1,64 \pm 0,07$ \\
Saison sèche & 100 & $1,54 \pm 0,05$ \\
\hline
\end{tabular}

Influence de l'âge:

L'analyse statistique ne montre pas de différence entre classes d'âge.

\begin{tabular}{|c|c|c|c|}
\hline \multicolumn{4}{|c|}{ TABLEAU $N^{\circ}$ XLVI } \\
\hline classe & d'âge & $\mathrm{N}$ & $\left\{\begin{array}{l}\text { Valeur moyenne } \pm \text { intervalle } \\
\text { de confiance à } 5 \text { p.100 }\end{array}\right.$ \\
\hline & $D$ & 26 & $1,21 \pm 0,14$ \\
\hline & E & 61 & $1,33 \pm 0,08$ \\
\hline & $\mathrm{F}$ & 37 & $1,30 \pm 0,12$ \\
\hline & G & 80 & $1,18 \pm 0,08$ \\
\hline & $\mathrm{H}$ & 15 & $1,27 \pm 0,21$ \\
\hline
\end{tabular}

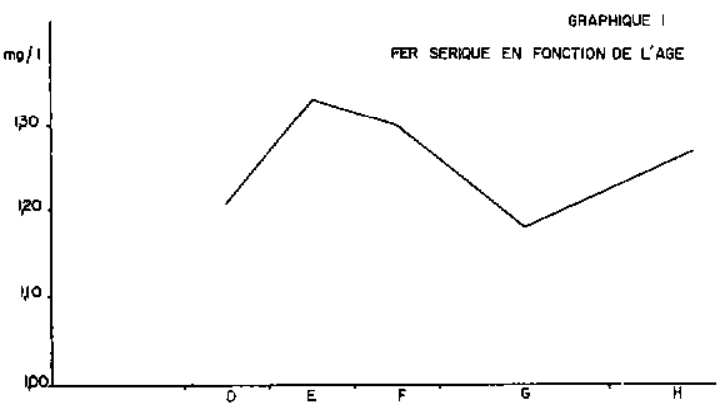




\section{Influence de l'espèce:}

Les résultats sont assez étonnants : les zébus ne diffèrent pas des taurins mais les métis sont très significativement différents de l'ensemble taurins - zébus.

TABLEAU N ${ }^{a}$ XLVII

Influence de 1 'espèce

\begin{tabular}{|l|c|c|}
\hline Espèce & $\mathrm{N}$ & $\begin{array}{c}\text { Valeur moyenne } \pm \text { interva11e } \\
\text { de confiance à } 5.100\end{array}$ \\
\hline Zébus & 89 & $1,18 \pm 0,06$ \\
Métis & 36 & $1,58 \pm 0,13$ \\
Taurins & 94 & $1,20 \pm 0,07$ \\
\hline
\end{tabular}

\section{LE SODIUM}

1687 analyses de sodium nous donnent une moyenne de $3401 \mathrm{mg} / \mathrm{l} \pm 15$. Les normes européennes varient de 3200 à $3800 \mathrm{mg} / \mathrm{l}$; la moyenne sénégalaise se situe dans ces normes.

\section{Influence de la région:}

La natrém e des bovins sénégalais varie d'une région à l'autre tout en restant dans les normes des pays européens.

Influence de la saison:

L'analyse de variance montre que la natrémie est indépendante de la saison.

TABLEAU N ${ }^{\circ} \mathrm{XLVIII}$

Influence de la région

\begin{tabular}{|l|c|c|}
\hline \multicolumn{1}{|c|}{ Région } & $\mathrm{N}$ & $\begin{array}{c}\text { Valeur moyenne } \pm \text { intervalle } \\
\text { de confiance } 5 \text { p.100 }\end{array}$ \\
\hline Ferlo & 485 & $3.224 \pm 15$ \\
Casamance & 142 & $3.329 \pm 30$ \\
C.R.Z. Dara & 240 & $3.185 \pm 22$ \\
Zone arachidière & 820 & $3.581 \pm 21$ \\
\hline
\end{tabular}

TABLEAU N* XLIX

Influence de la saison

\begin{tabular}{|l|c|c|}
\hline \multicolumn{1}{|c|}{ Saison } & N & $\begin{array}{c}\text { Valeur moyenne } \pm \text { intervalle } \\
\text { de confiance à 5 p.100 }\end{array}$ \\
\hline Hivernage & 292 & $3.489 \pm 35$ \\
Post hivernage & 375 & $3.487 \pm 37$ \\
Saison sèche & 393 & $3.497 \pm 30$ \\
\hline
\end{tabular}

Influence de l'âge :

La natrémie des bovins est fonction de l'âge. La classe $\mathrm{D}$ est différente de la classe $\mathrm{E}$ TABLEAU $\mathrm{N}^{*} \mathrm{~L}$

Influence de 1 'âge

\begin{tabular}{|c|c|c|}
\hline Classe d'âge & N & $\begin{array}{c}\text { Valeur moyenne } \pm \text { intervalle } \\
\text { de confiance a } 5 \text { p.100 }\end{array}$ \\
\hline D & 44 & $3.743 \pm 118$ \\
E & 88 & $3.607 \pm 50$ \\
F & 50 & $3.525 \pm 59$ \\
G & 96 & $3.548 \pm 48$ \\
H & 15 & $3.544 \pm 91$ \\
\hline
\end{tabular}

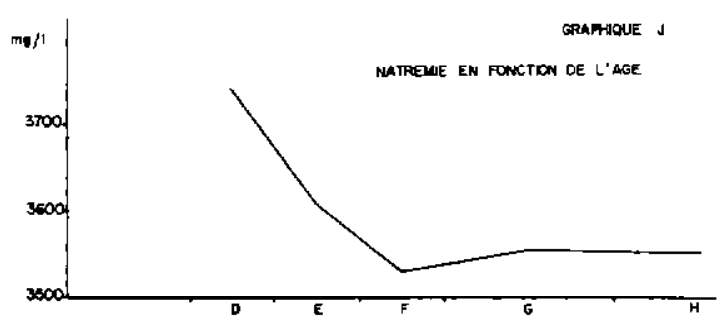


elle-même différente de l'ensemble $F, G, H$ qui forme un groupe homogène. La natrémie décroît en fonction de l'âge.

\section{Influence de l'espèce:}

Toutes les espèces sont significativement différentes les unes des autres.

TABLEAU $\mathrm{N}^{\circ} \mathrm{L} 1$

Influence de l'espèce

\begin{tabular}{|l|r|c|}
\hline Espèce & N & $\begin{array}{c}\text { Valeur moyenne } \pm \text { intervalle } \\
\text { de confiance a } 5 \text { p.100 }\end{array}$ \\
\hline Zébus & 164 & $3.679 \pm 43$ \\
Métis & 38 & $3.386 \pm 58$ \\
Taurins & 91 & $3.518 \pm 42$ \\
\hline
\end{tabular}

\section{LE POTASSIUM}

La moyenne générale sur 1667 données est de $198,2 \mathrm{mg} / 1 \pm 1,4$. Les moyennes européennes variant de 150 à $230 \mathrm{mg} / \mathrm{l}$, la moyenne sénégalaise se situe dans les normes.

\section{Influence de la région:}

Il existe des différences entre régions mais la kaliémie varie malgré tout très peu d'une région à l'autre. Les taux du Ferlo sont inférieurs aux taux de la Casamance et de la Zone arachidière.

\section{Influence de la saison:}

Il apparaît des différences significatives entre toutes les saisons. La kaliémie de saison sèche est plus élevée que celle du post hivernage, elle-même plus élevée que la kaliémie de l'hivernage.

TABLEAU N`LII

Influence de la région

\begin{tabular}{|l|c|c|}
\hline \multicolumn{1}{|c|}{ Région } & $\mathbf{N}$ & $\begin{array}{c}\text { Valeur moyenne } \pm \text { intervalle } \\
\text { de confiance à 5.100 }\end{array}$ \\
\hline Ferlo & 473 & $179 \pm 2,2$ \\
Casamance & 142 & $200,3 \pm 3,5$ \\
C.R.z. Dara & 240 & $184,0 \pm 1,6$ \\
Zone arachidière & 812 & $213,3 \pm 2,0$ \\
\hline
\end{tabular}

TABLEAU $N^{0}$ LIII

Influence de la saison

\begin{tabular}{|l|c|c|}
\hline \multicolumn{1}{|c|}{ Saison } & N & $\begin{array}{c}\text { Valeur moyenne } \pm \text { intervalle } \\
\text { de confiance à 5 p.100 }\end{array}$ \\
\hline Hivernage & 292 & $194,7 \pm 2,5$ \\
Pogt hivernage & 337 & $208,5 \pm 3,0$ \\
Saison sèche & 423 & $213,3 \pm 2,9$ \\
\hline
\end{tabular}

\section{Influence de l'âge :}

La kaliémie des animaux dépend de l'âge. Nous pouvons regrouper les classes $D$ et $E$ ainsi que les classes F, G, H. La kaliémie des jeunes jusqu'à 4 ans est significativement différente de celle des adultes.

\section{Influence de l'espèce:}

La kaliémie varie beaucoup d'une espèce à l'autre. Le taux de potassium sérique est le plus élevé chez les zébus. 


TABLEAU $N^{*}$ LIV
Influence de 1 'âge
\begin{tabular}{|c|r|r|}
\hline Classe d'âge & iv & $\begin{array}{c}\text { Valeur moyenne } \pm \text { intervalle } \\
\text { de confiance à } 5 \text { p.100 }\end{array}$ \\
\hline D & 46 & $233,7 \pm 9,1$ \\
E & 89 & $226,7 \pm 7,1$ \\
F & 53 & $216,2 \pm 8,8$ \\
G & 100 & $215,8 \pm 5,7$ \\
H & 15 & $217,3 \pm 21,6$ \\
\hline
\end{tabular}

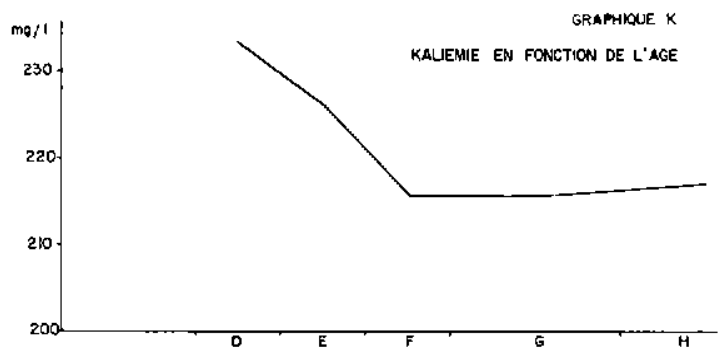

TABLEAU $\mathrm{N}^{*} \mathrm{LV}$

Influence de 1 'espèce

\begin{tabular}{|l|r|c|}
\hline \multicolumn{1}{|c|}{ Espèce } & N & $\begin{array}{c}\text { Valeur moyenne } \pm \text { incervalle } \\
\text { de confiance à 5 p.100 }\end{array}$ \\
\hline Zëbus & 171 & $237,6 \pm 4,4$ \\
Mêtis & 38 & $187,1 \pm 5,4$ \\
Taurins & 94 & $207,0 \pm 4,4$ \\
\hline
\end{tabular}

\section{LE MAGNESIUM}

Nous avons trouvé une moyenne générale de $26,4 \mathrm{mg} / 1 \pm 0,3$ sur 1617 analyses effectuées Cette moyenne dépasse la norme européenne qui est d'environ $22 \mathrm{mg} / \mathrm{l}$.

Influence de la région:

La magnésémie varie d'une région à l'autre.

Influence de la saison:

Il existe des différences entre saisons en ce qui concerne les taux de magnésium sérique. Le taux du post hivernage est le plus près du taux moyen européen. Là encore nous retrouvons l'effet bénéfique du post hivernage.

TABLEAU $\mathrm{N}^{*}$ LVI

Influence de la région

\begin{tabular}{|c|c|c|}
\hline Région & $\mathrm{N}$ & $\begin{array}{l}\text { Valeur moyenne } \pm \text { intervalle } \\
\text { de confiance à } 5 \text { p.100 }\end{array}$ \\
\hline Fer 10 & $4 B 4$ & $22,8 \pm 0,4$ \\
\hline Casamance & 141 & $25,7 \pm 1,1$ \\
\hline C.R.Z. Dara & 240 & $26,4 \pm 0,4$ \\
\hline Zone arachidière & 752 & $28,8 \pm 0,4$. \\
\hline
\end{tabular}

TABLEAU $\mathrm{N}^{*}$ LVII

Influence de la saison

\begin{tabular}{|c|c|c|}
\hline Saison & N & $\begin{array}{l}\text { Valeur moyenne } \pm \text { intervalle } \\
\text { de confiance à } 5 \mathrm{p} .100\end{array}$ \\
\hline Hivernage & 291 & $29,0 \pm 0,6$ \\
\hline Post hivernage & 318 & $25,8 \pm 0,6$ \\
\hline Saison sèche & 383 & $29,5 \pm 0,4$ \\
\hline
\end{tabular}

\section{Influence de l'âge:}

L'analyse de variance ne montre pas de diifférence entre les classes d'âge (tableau LVIII et graphique L).

\section{Influence de l'espèce :}

Il n'existe pas de différence entre zébus et taurins mais des différences hautement significatives apparaissent entre métis-taurins et entre métis-zébus (tableau LIX). 
TABLEAU N ${ }^{*}$ LVIII

Influence de l'âge

\begin{tabular}{|c|r|c|}
\hline Classe d'âge & $N$ & $\begin{array}{c}\text { Valeur moyenne } \pm \text { intervalle } \\
\text { de confiance a } 5.100\end{array}$ \\
\hline D & 45 & $31,0 \pm 1,3$ \\
E & 81 & $30,1 \pm 0,9$ \\
F & 50 & $31,0 \pm 1,1$ \\
G & 93 & $30,4 \pm 0,9$ \\
H & 14 & $29,7 \pm 2,1$ \\
\hline
\end{tabular}

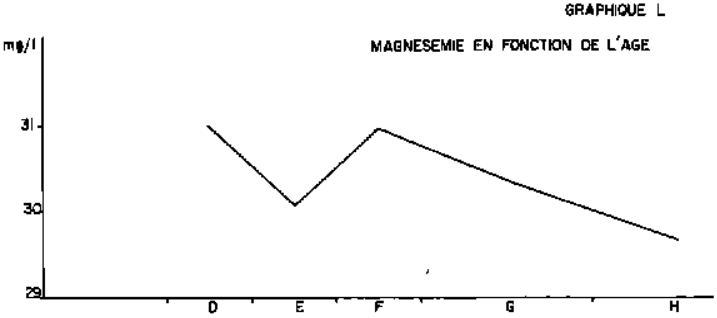

TABLEAU $\mathrm{N}^{\circ}$ LIX

Influence de l'espèce

\begin{tabular}{|l|r|c|}
\hline \multicolumn{1}{|c|}{ Espèce } & N & $\begin{array}{c}\text { Valeur moyenne } \pm \text { interva1le } \\
\text { de confiance à } 5 \text { p.100 }\end{array}$ \\
\hline Zébus & 153 & $31,2 \pm 0,6$ \\
Métis & 38 & $27,8 \pm 1,5$ \\
Taurins & 92 & $30,3 \pm 0,9$ \\
\hline
\end{tabular}

\section{DISCUSSIONS ET CONCLUSIONS CONCERNANT CES RESULTATS}

L'objectif de ce travail statistique et des longues énumérations auxquelles il a donné lieu était donc d'établir des normes sénégalaises concernant 12 éléments sériques à partir de plus de 20000 dosages biochimiques effectués ces dernières années. Cet objectif a été réalisé en partie, comme en témoigne le tableau général LX des résultats présentés ci-contre.

Ces résultats généraux qui présentent un haut degré de probabilité en raison du grand nombre d'analyses ont été décomposés en résultats partiels tenant compte des divers facteurs de variations contrôlables qui sont la région, la saison, l'âge, le sexe, l'espèce. (Nous n'avons pu envisager dans cette étude un facteur important pour les femelles, celui qui intéresse l'état physiologique de reproduction.)

Mais au-delà de cette recherche des normes, indispensable pour juger de tous les cas particuliers, ce travail a permis d'effectuer un certain nombre de remarques que nous allons maintenant présenter.

Il faut d'abord conserver à l'esprit que les conclusions qu'on peut tirer d'une analyse biochimique ne sont pas immédiates, mais doivent être discutées et replacées dans un contexte. La composition du sang fait partie d'une de ces homéostasies premières à laquelle participent à la fois le milieu extérieur et tous les mécanismes de régulation de l'organisme.

Une déviation quelconque dans cette constance peut résulter de nombreux processus dont il convient chaque fois de pondérer l'importance relative.

Quelques exemples caractéristiques montrent bien les difficultés d'une interprétation biochimique. Si l'on considère le taux des protéines par exemple, on voit que ce ne sont pas les valeurs les plus élevées qui correspondent à l'état physiologique le plus favorable, qui semble se situer au niveau des normes européennes $(76 \mathrm{~g} / 1)$. On constate en effet que le troupeau sénégalais abaisse ses normes vers ces valeurs dans les saisons favorables (le post hivernage), et dans les régions où en général les ressources alimentaires sont plus abondantes et plus équilibrées. (Zone arachidière par rapport au Ferlo.) Sur le taux des protéines, on peut donc distinguer deux facteurs d'origine différente : le facteur extérieur qui, au Ferlo par exemple, en raison de la 
T A B L E A U $\mathbb{N}^{0}$ LX

Normes de 12 paramétres biochimiques du sang chez les bovins du sénégal

\begin{tabular}{|c|c|c|c|c|c|c|c|c|c|c|c|c|c|}
\hline & & Hếma tocrite & $\begin{array}{c}\text { Hêmoglobine } \\
\mathrm{g} / 100\end{array}$ & $\begin{array}{l}\text { Protéines } \\
\mathrm{g} / \mathrm{I}\end{array}$ & $\begin{array}{l}\text { Urée } \\
\mathrm{mg} / 1\end{array}$ & $\begin{array}{l}\text { Phosphore } \\
\mathrm{mg} / 1\end{array}$ & $\begin{array}{l}\text { Calcium } \\
\mathrm{mg} / 1\end{array}$ & $\begin{array}{c}\text { Cuivre } \\
\mathrm{mg} / 1\end{array}$ & $\begin{array}{l}\operatorname{Linc} \\
\mathrm{mg} / 1\end{array}$ & $\begin{array}{l}\mathrm{Fer} \\
\mathrm{mg} / 1\end{array}$ & $\begin{array}{l}\text { Sodium } \\
\mathrm{mg} / 1\end{array}$ & $\begin{array}{l}\text { Potassium } \\
\text { mg/1 }\end{array}$ & $\begin{array}{c}\text { Magnésium } \\
\text { mg/1 }\end{array}$ \\
\hline \multicolumn{2}{|c|}{ Moyenne genérale } & $39,1 \pm 0,3$ & $11,5 \pm 0,1$ & $85,4 \pm 0,8$ & $293 \pm 6$ & $62,1 \pm 0,9$ & $102,6 \pm 0,6$ & $0,60 \pm 0,01$ & $2,46 \pm 0,03$ & $1,32 \pm 0,03$ & $3401 \pm 15$ & $198,2 \pm 1,4$ & $26,4 \pm 0,3$ \\
\hline \multirow{4}{*}{ Région } & Ferlo & $38,8 \pm 0,4$ & $12,0 \pm 0,1$ & $84,2 \pm 1,0$ & $268 \pm 6$ & $50,1 \pm 1,4$ & $102,6 \pm 0,8$ & $0,55 \pm 0,01$ & $1,50 \pm 0,05$ & $1,17 \pm 0,04$ & $3224 \pm 15$ & $179,0 \pm 2,2$ & $22,8 \pm 0,4$ \\
\hline & Dara (C.R.Z.) & $44,2 \pm 0,7$ & & $80,2 \pm 1,3$ & & $74,5 \pm 1,4$ & $96,5 \pm 0,9$ & $0,60 \pm 0,01$ & $1,31 \pm 0,03$ & $1,59 \pm 0,03$ & $3185 \pm 22$ & $184,0 \pm 1,6$ & $26,4 \pm 0,4$ \\
\hline & Zone arachidière & $38,0 \pm 0,4$ & $11,1 \pm 0,1$ & $87,9 \pm 1,4$ & $313 \pm 9$ & $73,3 \pm 1,1$ & $105,4 \pm 1,3$ & $0,69 \pm 0,01$ & $1,39 \pm 0,03$ & $1,25 \pm 0,04$ & $3581 \pm 21$ & $213,3 \pm 2$ & $28,8 \pm 0,4$ \\
\hline & $\begin{array}{l}\text { Sangalkam } \\
\text { (embouche) }\end{array}$ & $44,1 \pm 0,3$ & $11,8 \pm 0,5$ & $84,3 \pm 1,5$ & $393 \pm 29$ & $90,3 \pm 2,4$ & $90,8 \pm 1,3$ & $0,59 \pm 0,02$ & $1,04 \pm 0,04$ & & & & \\
\hline \multirow{3}{*}{$\begin{array}{l}\text { Saisons } \\
\text { Dara } 70+ \\
\text { (Zone ara- } \\
\text { chidière) }\end{array}$} & Hivernage & $39,2 \pm 0,6$ & $11,3 \pm 0,2$ & $101,3 \pm 3,0$ & $384 \pm 15$ & $64,9 \pm 2,0$ & $109,9 \pm 1,3$ & $0,63 \pm 0,02$ & $1,41 \pm 0,05$ & $1,63 \pm 0,06$ & $3489 \pm 35$ & $194,7 \pm 2,5$ & $29,0 \pm 0,6$ \\
\hline & Post-hivernage & $39,9 \pm 0,5$ & $10,9 \pm 0,2$ & $81,5 \pm 0,9$ & $240 \pm 10$ & Fer10 67 & $89,3 \pm 2,0$ & $0,75 \pm 0,02$ & $1,30 \pm 0,07$ & $1,64 \pm 0,07$ & $3487 \pm 37$ & $208,5 \pm 3,0$ & $25,8 \pm 0,6$ \\
\hline & Saison sèche & $38,4 \pm 0,7$ & $1,1,2 \pm 0,2$ & $81,9 \pm 1,9$ & $367 \pm 18$ & $50,7 \pm 2,0$ & $107,5 \pm 1,4$ & $0,61 \pm 0,02$ & $1,26 \pm 0,06$ & $1,54 \pm 0,05$ & $3497 \pm 30$ & $213,3 \pm 2,9$ & $29,5 \pm 0,4$ \\
\hline \multirow{5}{*}{$\begin{array}{l}\text { Age } \\
\text { Labgar } 72+ \\
\text { (Zone ara- } \\
\text { ch1d1ëre } 72 \text { ) }\end{array}$} & $0 \grave{\mathrm{a}} 2$ ans & $38,0 \pm 1,3$ & $11,7 \pm 0,4$ & $80,0 \pm 3,8$ & $300 \pm 23$ & $38,7 \pm 3,9$ & $111,9 \pm 3,1$ & $0,62 \pm 0,04$ & $1,39 \pm 0,12$ & $1,21 \pm 0,14$ & $3743 \pm 118$ & $233,7 \pm 9,1$ & $31,0 \pm 1,3$ \\
\hline & 2 à 4 ans & $40,6 \pm 0,7$ & $12,4 \pm 0,2$ & $86,0 \pm 2,5$ & $307 \pm 16$ & $37,1 \pm 1,7$ & $109,1 \pm 1,5$ & $0,58 \pm 0,02$ & $1,31 \pm 0,05$ & $1,33 \pm 0,08$ & $3607 \pm 50$ & $226,7 \pm 7,1$ & $30,1 \pm 0,9$ \\
\hline & 4 a 6 ans & $37,5 \pm 0,9$ & $11,7 \pm 0,3$ & $93,1 \pm 2,7$ & $309 \pm 23$ & $29,3 \pm 2,7$ & $105,0 \pm 2,0$ & $0,54 \pm 0,02$ & $1,29 \pm 0,06$ & $1,30 \pm 0,12$ & $3525 \pm 59$ & $216,2 \pm 8,8$ & $31,0 \pm 1,1$ \\
\hline & 6 à 10 ans & $36,0 \pm 0,7$ & $11,1 \pm 0,3$ & $89,9 \pm 2,3$ & $300 \pm 15$ & $28,8 \pm 2,0$ & $104,8 \pm 1,9$ & $0,54 \pm 0,02$ & $1,25 \pm 0,05$ & $1,18 \pm 0,08$ & $3548 \pm 48$ & $215,8 \pm 5,7$ & $30,4 \pm 0,9$ \\
\hline & $>\quad 10$ ans & $35,8 \pm 1,4$ & $11,2 \pm 0,5$ & $94,4 \pm 5,9$ & $286 \pm 29$ & $29,2 \pm 4,2$ & $104,1 \pm 3,0$ & $0,51 \pm 0,03$ & $1,21 \pm 0,07$ & $1,27 \pm 0,24$ & $3544 \pm 91$ & $217,3 \pm 21,6$ & $29,7 \pm 2,1$ \\
\hline \multirow{3}{*}{$\begin{array}{l}\text { Sexe } \\
\text { (Labgar 72) }\end{array}$} & Femelles & $38,3 \pm 0,6$ & $11,9 \pm 0,2$ & $92,0 \pm 1,5$ & $259 \pm 8$ & $30,6 \pm 1,3$ & $102,3 \pm 1,2$ & $0,51 \pm 0,03$ & $1,22 \pm 0,04$ & & & & \\
\hline & Mâ1es & $38,5 \pm 1,5$ & $12,0 \pm 0,6$ & $87,7 \pm 4,0$ & $265 \pm 21$ & $37,9 \pm 2,5$ & $107,0 \pm 3,3$ & $0,53 \pm 0,05$ & $1,21 \pm 0,08$ & & & & \\
\hline & Castrés & $42,4 \pm 1,8$ & $12,8 \pm 0,6$ & $90,8 \pm 5,1$ & $270 \pm 22$ & $39,9 \pm 2,8$ & $108,6 \pm 3,3$ & $0,53 \pm 0,04$ & $1,18 \pm 0,07$ & & & & \\
\hline \multirow{3}{*}{$\begin{array}{l}\text { Espèce } \\
\text { (Zone ara- } \\
\text { chidiēre } 72 \text { ) }\end{array}$} & Zébus & $37,5 \pm 0,9$ & $11,6 \pm 0,3$ & $85,9 \pm 3,9$ & $408 \pm 26$ & $80,6 \pm 2,6$ & $114,1 \pm 2,1$ & $0,68 \pm 0,02$ & $1,80 \pm 0,25$ & $1,18 \pm 0,06$ & $3679 \pm 43$ & $237,6 \pm 4,4$ & $31,2 \pm 0,6$ \\
\hline & Métis & $37,7 \pm 1,2$ & $10,5 \pm 0,4$ & $72,3 \pm 2,4$ & $358 \pm 42$ & $81,5 \pm 6,3$ & $107,6 \pm 3,1$ & $0,54 \pm 0,04$ & $1,52 \pm 0,25$ & $1,58 \pm 0,13$ & $3386 \pm 58$ & $187,1 \pm 5,4$ & $27,8 \pm 1,5$ \\
\hline & Taurins & $34,7 \pm 1,2$ & $11,1 \pm 0,6$ & $82,9 \pm 3,2$ & $301 \pm 24$ & $66,7 \pm 1,8$ & $109,3 \pm 2,9$ & $0,54 \pm 0,03$ & $1,46 \pm 0,12$ & $1,20 \pm 0,07$ & $3518 \pm 42$ & $207,0 \pm 4,4$ & $30,3 \pm 0,9$ \\
\hline
\end{tabular}

Addendum: Colonne «Phosphore», au lieu de « Ferlo 67 \%, lire « Ferlo 67 \%. 
pauvreté en azote des pailles de saison sèche fait que le taux des protéines sériques baisse durant cette période; un facteur interne lié à l'état physiologique de l'individu. En hivernage par exemple, les animaux qui baignent dans un milieu devenu brutalement excédentaire en azote stockent au maximum ce précieux élément. Le taux des protéines circulantes s'élève alors. Durant le post hivernage, au stockage désorganisé va succéder une phase de métabolisation. L'azote est introduit dans les structures de l'individu qui opposeront une résistance plus efficace au catabolisme de saison sèche.

Le problème est encore plus complexe quand il s'agit du phosphore par exemple. Très tôt après la fin de l'hivernage, les apports alimentaires deviennent insuffisants et on assiste à une chute lente et progressive de la phosphorémie étroitement bridée par les mécanismes hormonaux (parathyrö̈diens en particulier). Mais si ces mécanismes viennent à être débordés la situation s'inverse et les individus, faisant preuve d'un épuisement extrême ou parvenus à la phase agonique, révèlent une phosphorémie anormalement élevée.

Une donnée d'interprétation encore plus délicate est l'urémie qui fait intervenir deux facteurs extérieurs (apport azoté de la ration, équilibre énergie azote de la ration) et de multiples facteurs internes (activité de la flore bactérienne, mécanisme d'économie au niveau du rein, etc.).

En définitive, ces exemples soulignent parfaitement la difficulté des interprétations biochimiques qui ont besoin pour devenir fructueuses d'être discutées en fonction du contexte. Ce contexte, et ce sera là notre conclusion, s'inscrit dans la trilogie suivante : agrostologie, bromatologie et digestibilité-biochimie.

En effet, au Sénégal et en zone intertropicale en général, le milieu naturel est à la fois le support et le pourvoyeur de nourriture exclusif de la plus grande partie du cheptel. La connaissance de ce milieu naturel, dominé par des conditions climatologiques très dures, est encore très imparfaite.

Beaucoup de ces incertitudes pourraient être levées si partout les trois disciplines évoquées progressaient en étroite coordination suivant un schéma rationnel qui pourrait être le suivant :

Etude de base par l'agrostologie, complétée par l'appréciation de la valeur de l'aliment végétal, l'animal servant alors de révélateur pour confirmer ou infirmer les deux démarches précédentes.

\section{SUMMARY \\ Biochemistry and breeding in Senegal}

The present study tries to establish biochemical standards for 12 components of bovine blood cattle in Senegal. From 24605 analyses values of hematocrit, haemoglobin, proteins, urea, phosphorus, calcium, magnesium, sodium, potassium, cupper, iron and zinc are determined. The effect of region, age, sex, season and breed is studied for each element. Results are compared to European averages and to results obtained in intensive fattening experiments in Senegal.

\section{RESUMEN}

\section{Bioquimica y ganaderia en Senegal}

Los autores intentaron de establecer normas bioquimicas por 12 componentes de la sangre de los bovinos en Senegal. Determinaron a partir de 24605 analisis los valores del hematocrito, hemoglobina, proteínas totales, urea, fósforo, calcio, magnesio, sodio, potasio, cobre, hierro y cinc. Se estudia con cada elemento la influencia de la región, de la estación, de la edad, del sexo y de la raza.

Se comparan los resultados con las tasas medias európeas y con las obtenidas durante ensayos de engorde en Senegal. 


\section{BIBLIOGRAPHIE}

1. ARCHAMBAULT de VENCAY (J.), CALVET (H.), BOUDERGUES (R.). Recherches sur le métabolisme du rumen chez les bovins tropicaux (3e partie). Rev. Elev. Méd. vét. Pays trop., 1971, 24 (2): 307-312.

2. BOUDERGUES (R.), CALVET (H.). Protéinogramme des sérums de zébus Gobra au Sénégal. Variations quantitatives saisonnières. Rev. Elev. Méd. vét. Pays trop., 1971, 24 (4) : 581-586.

3. BOUDERGUES (R.), CALVET (H.), ARCHAMBAULT de VENCAY (J.). Recherches sur le métabolisme du rumen chez les bovins tropicaux (2e partie). Rev. Elev. Méd. vét. Pays trop., 1971, 24 (2): 297-305.

4. CALVET (H.), PICART (P.), DOUTRE (P.M.). CHAMBRON (J.). Aphosphorose et botulisme au Sénégal. Rev. Elev. Méd. vér. Pays trop., 1965, 18 (5): 249-282.

5. CALVET (H.), FRIOT (D.) et CHAMBON (J.). Influence des supplémentations minérales sur le croît et sur certains témoins biochimiques du métabolisme minéral chez les bovins tropicaux. Rev. Elev. Méd. vét. Pays trop., 1972, 25 (3) : 397-408.

6. CALVET (H.), BOUDERGUES (R.), REMISY (C.) et ARCHAMBAULT de VENCAY (J.). Recherches sur le métabolisme du rumen chez les bovins tropicaux (1're partie). Rev. Elev. Méd, vét. Pays trop., 1971, 24 (2) : 287-296.

7. CALVET (H.), ARCHAMBAULT de VENCAY (J.) et BOUDERGUES (R.). Recherches sur le métabolisme du rumen chez les bovins tropicaux (4e partie). Rev. Elev. Méd. vét. Pays trop., 1971, 24 (2): 313-318.

8. CHARPENTIER (J.) et BONHOMME (D.). Facteurs de variations de l'hématocrite des bovins. Ann. Zootechn., 1968, 17 (3) : 327-335.

9. DUKES. Physiology of domestic animals. London, Baillière, Tindall and Cox, 1955.

10. FERRANDO (R.). Profils biochimiques, sémiologie et élevage moderne. Cah. Méd. vét., 1971, 40: 47-56.

11. FRIOT (D.), CALVET (H.). Etude complémentaire sur les carences minérales rencontrées dans les troupeaux du Nord Sénégal. Rev. Elev. Méd. vét. Pays trop., 1971, 24 (3) : 393-407.

12. LABOUCHE (C.). Contribution à la connaissance du transit de l'urée chez les ruminants. Recherches sur l'urémie et l'élimination rénale de l'urée chez les bovins domestiques en milieu tropical. Thèse Sciences, Toulouse, 1967, no 306.

13. LABOUCHE (C.). La protéinémie chez la vache. Rev. Elev. Méd. vét. Pays trop., 1964, 17 (4) : $721-745$.

14. LABOUCHE (C.), AMALOU (P.) et CALVET (H.). Variations physiologiques de l'albumine sérique chez la vache adulte en milieu tropical. C.R. Soc. Biol., 1963, 157: 1784-1786.

15. LABOUCHE (C.), AMALOU (P.) et CALVET (H.). Variations physiologiques de l'albumine sérique chez la vache adulte en milieu tropical. C.R. Soc. Biol., 1963, 157: 1472-1475.

16. LABOUCHE (C.) et AMALOU (P.). Variations physiologiques des protéines totales du sérum de vache adulte en milieu tropical. C.R. Soc. Biol., 1963, 157: 604-609.

17. PICART (P.), CALVET (H.). Mesure des compartiments liquidiens corporels chez des bovins de l'Ouest africain. Rev. Elev, Méd. vét. Pays trop., 1967, 20 (2) : 311-327. 